\title{
RECHERCHE COMPARÉE DU CORPUS LEXICAL DES RÉSIDENTS DE LANGUE MATERNELLE HONGROISE DANS DEUX RÉGIONS DIFFÉRENTS
}

\section{Introduction}

A Szombathely, ville de Hongrie occidentale à la chaire de linguistique hongroise de l'École Normale Supérieure "Berzsenyi Dániel", on fait des recherches de géographie linguistique et sociolinguistiques depuis 1973 sous la conduite de Géza Szabó (Szabó, G. 1976). Moi, je me suis associé à ces recherches en 1978. Avant, jusqu'à 1984, j'ai étudié l'usage de langue des normaliens de Szombathely, donc des étudiants provenant en grande partie de Transdanubie Occidentale (Molnár 1980). Pendant ce temps, je me suis appliqué à connaître le plus minutieusement possible le terrain dialectal en question (Molnár 1990). En même temps j'ai eu la possibilité de me faire une idée sur l'usage de langue des Hongrois vivant dans les pays voisins, surtout des Hongrois de Slovénie entre 1988 et 1993 en qualité de lecteur à Maribor (Molnár 1986, 1991).

Toutes les deux recherches, donc celle des alentours de Szombathely et celle de la région de Lendva (en slovène Lendava) sont séparément riches en enseignements. Mais leur comparaison offre également des conclusions. Dans mon étude j'essaie de signaler quelques-unes de ces conclusions.

Pour le fond de perspective et pour les principes méthodologiques de la recherche j'ai essayé de trouver des points de repère également dans la littérature de spécialité nationale et internationale. J'ai à ma disposition beaucoup de bons modèles précédents et contemporains se rapportant sur la pratique de la sociolinguistique basée sur la géographie linguistique, sur le rapport de la collecte de mots et de textes par des questionnaires ou par magnétophone et pour leur dépouillement d'un aspect nuancé. Le plus directement je me sers des recherches, des matériaux auxiliaires et des études de Géza Szabó mentionné ci-dessus. Les recherches spécifiques de la collectivité de travail de sa chaire qui visent par exemple la conscience de la langue parlée, le comportement, la situation communicative, le milieu, le contact, ets. sont également riches en enseignements (voir p. e. BDTF TudKözl. I, 131-47; IV, 49-90; V, 9-19; VI, 5-87; DialSzimp. I, 55-77; 171-8; II, 47-54, 79-112, 163-72). 
De la dialectologie générale hongroise contemporaine dans une large acception pour moi sont importantes les méthodes qui décrivent ou qui comparent les dialectes locaux ou régionaux (voir p. e. à part les volumes de DialSzimp. mentionnés ci-dessus MNyRétegz.). Je m'adresse avec un intérêt spécial aux valeurs éternellement classiques de MNyA., au caractère du substrat dialectal et à ses conseils théoriquesméthodologiques qui stimulent aussi à une comparaison dialecte-langue courante (voir la littérature de spécialité relative à MNyA., surtout MNyAElmMódsz.).

Étant donné que dans la préparation et dans l'élaboration de l'Atlas linguistique de la langue hongroise - qui est internationalement reconnu - un grand rôle a eu une large documentation étrangère (conf. l'ouvrage cité 31,51-63, etc.), d'une manière indirecte on peut assister à l'apparition européenne de l'idée de la géographie linguistique. Dans ce domaine - découlant aussi de mes possibilités - c'étaient les rapports français, allemands, italiens, russes et slovènes qui ont éveillé mon intérêt, mais les publications hongroises rendent possible un aperçu sur la littérature de spécialité linguistique anglaise, finnoise, etc., et par cela sur un domaine plus étendu (Kálmán 1983, 1986; Kiss 1987; etc.). La littérature internationale de géographie linguistique et de recherches de langue régionale, sa valeur culturelle, son caractère national, ses méthodes nuancées, et à travers tous ces aspects, la continuation, le renouvellement et l'élargissement de la géographie linguistique classique (p. e. Goebl 1988. 76; Jernej 1988; Korotkova 1988. 52, 53, 58, 59; Kovačec 1992. 159, 170, 171, 172).

Mon activité de Maribor mentionnée ci-dessus m'a permis, surtout dans le dernier temps, de connaître la situation slovène. En Slovénie, la géographie linguistique a de riches traditions. Certainement ce n'est pas indépendant du fait que dans ce petit pays ayant une population en tout de deux millions - à cause de ses caractéristiques géographiques, historiques, etc. - la répartition dialectale est prononcée (voir Logar 1975, 1989. 12; Jakopin 1989. 61). Il y avait et il y a de nos jours aussi une exigence pour la description des variantes locales et territoriales par la méthode atlas ou avec un annexe atlas (Tesnière 1925; Ramovš 1931; FonOpisi 27-218; SLA; etc.). De la littérature specialisée slovène contemporaine me paraissent instructifs surtout les procédés qui tendent à la description la plus nuancée des dialectes (voir Zorko 1989 a, b). Ces procédés étudient les dialectes dans l'unité avec les autres variantes de la langue slovène (p.e. Toporišič 1991) ou ils accordent une grande importance aux rapports sociolinguistiques dans la recherche de la communication linguistique (p. e. Križman 1989; Nečak Lük 1992). Les recherches en Slovénie sont également intéressantes parce qu'elles permettent de connaitre la recherche des dialectes de cette langue qui se trouve à la lisière occidentale du territoire linguistique slave ayant des contacts avec l'allemand et l'italien. Cela revient à dire que la situation de ces formations linguistiques est similaire à la situation des dialectes occidentaux du territoire linguistique hongrois qui se trouvent eux-aussi dans la proximité du territoire linguistique allemand et slave. 
Dans les expériences détaillées ci-dessus de la littérature specialisée slovène et autres j'ai le courage d'appliquer comparativement les aspects de géographie linguistique et sociolinguistiques dans la description dialectale.

\section{Mobiles théoriques et approche méthodologique}

\subsection{Le cadre théorique de la recherche}

La recherche actuelle essaie - en vertu de l'orientation présentée dans l'introduction - de définir une possibilité d'intérêt sociolinguistique de la dialectologie contemporaine. Cette recherche suppose que dans les différents points géographiques d'usage de la langue on peut surprendre des combinaisons particulières des traditions linguistiques locales (du dialecte) et de la langue standard (de la langue courante). Dans l'evolution de ces formes d'usage de la langue les régions plus ou moins grandes et les différentes relations sociales des résidants jouent un rôle important.

De cette manière la recherche suggère les questions fondamentales suivantes: Comment les relations de géographie linguistique se modifient-elles de nos jours dans certains points de l'ouest de l'usage de la langue hongroise? Comment sont les caractères sociolinguistiques dans ces cadres donnés? De quelle grandeur sont dans ces endroits l'unité et la diversification de la langue? Mon étude a donc avant tout un caractère dialectologique puisqu'elle se charge de faire la description de géographie linguistique contemporaine et de signaler la formation des régions par la démonstration des similitudes et des différences linguistiques. En même temps - élargissant le cadre de la recherche dans le domaine sociolinguistique - elle essaie de surprendre le côté linguistique de la stratification sociale des résidants de cette (ces) région(s).

D'une part, je présuppose l'existence des régions de langue en question à la suite des facteurs socioculturels (d'histoire de la civilisation, ethnographiques, sociologiques, etc.), et, d'autre part, je me base sur les remarques des recherches de la langue régionale courante concernant la condition linguistique de la ville et de ces environs (je parlerai encore de cette dernière dans la partie 2. 2. en abordant le choix des points de recherche). Mes propres recherches voudraient également confirmer l'existence de ces régions.

Les aspects sociolinguistiques voudraient examiner la validité de la thèse - selon laquelle la présence accrue des éléments du dialecte ou de la langue courante serait en corrélation avec la stratification âgé-jeune, homme-femme, moins exercé-cultivé. La région située en Hongrie rend acceptable de toute manière cette thèse, mais est-elle valable - si oui, en quelle mesure - pour la région non-hongroise? (Voir p. e. Szépe 1985. 270.)

Le corpus collecté par une méthodologie identique dans les deux régions distinctes rend possible aussi la comparaison. Bien qu'elles aient des conditions socioculturelles différentes, plusieurs raisons permettent leur analyse contrastive. Tant dans la région de 
Szombathely qu'aux alentours de Lendva, l'usage de la langue hongroise est une manifestation de langue maternelle. Toutes les deux font partie du type de dialecte hongrois de l'ouest (voir aussi le chapitre 2. 2.). Chacune est une formation régionale, celle de la région de Szombathely est la variation de la langue hongroise de Hongrie, tandis que celle des environs de Lendva est la variation de Slovénie de la même langue (conf. p. e. Lanstyák 1995. 119, 216-217; Deme 1995. 360, 364).

\subsection{L'échantillon}

De mon territoire de collecte je possède plus de 70 heures d'enregistrement de magnétophone et une notation d'environ 200 pages de données et de textes. Pour la présente recherche concrète j'ai choisi de la région de Szombathely comme de celle de Lendva aussi trois points d'enquête, notamment Köszegdoroszló (I.), Dozmat (II.) et Sorokpolány (III.), puis Hosszúfalu (Dolga vas - IV.), Csente (Čentiba - V.) et Felsôlakos (Gornji Lakoš -VI.). Les localités entourent en forme triangulaire les deux villes en question dont elles se situent - comme d'un centre - à une distance moyenne de 12, respectivement $3 \mathrm{~km}$ (voir la carte annexe; conf. Vas megye, 1979; Zbornik, 1981).

Une de ces localités, Kőszegdoroszló (I.) est située au nord de Szombathely, au pied du prolongement oriental des Alpes, dans une région spécifique nommée Kôszeghegyalja. Le nombre de ses habitants: 285 (KSH 152). Dozmat (II.) se trouve à l'ouest de Szombathely, cachée entre les collines marquant le début de la région des Alpes. Elle a 179 habitants (idem 151). Sorokpolány (III.) est une commune située au sud de Szombathely, dans une région relativement plate, non loin de la rivière Ràba ayant une population de 780 habitants (idem 153).

Hosszúfalu (IV.) en Slovénie est une localité de la région historique Hetés qui se trouve au nord de Lendva, près du mont Lendva. Elle a 763 habitants dont 454 sont hongrois (conf. Varga S. 1992. 87). Csente (V.) se trouve au sud-est de Lendva, au pied du mont Lendva, dans une vallée, le nombre de ses habitants est 778 dont 498 hongrois (voir le dernier ouvrage cité). Felsôlakos (VI.), au contraire, se trouve dans un site plus ouvert, dans la proximité de la rivière Mura, près d'une route battue. De ses 479 habitants 372 sont hongrois (idem).

Le choix des points d'enquête a été déterminé aussi par un certain hasard parce que ces points - surtout de la région de Mura - ont été choisis selon les lieux d'origine de mes étudiants qui m'ont servi d'intermédiaires dans la collecte du corpus. Mais de l'autre côté, la détermination du lieu de la recherche a été soutenue par des raisons fortes. L'un de ces motifs a été la présentation de la région linguistique de Szombathely dans les études de Géza Szabó et de sa chaire (voir leurs ouvrages cités). Le rapport linguistique des villes et de leurs environs a été déjà soulevé dans la matière de MNyA. (MNyAElmMódsz. 58, 59), puis il a été encore plus mis en relief dans la recherche de la langue courante régionale (Deme 1968). L'unité relative et la stratification en petites parties de la région de Szombathely ont été confirmées aussi par mes études préalables (Molnár 1989. 147-8). Quant au rapport de Lendva et de ses environs, mon attention a été attirée aussi par les recherches d'histoire de la civilisation, ethnographiques, 
linguistiques (Szentmihályi 1977. 70, 71; Varga J. 1985) et par les études analysant le rapport des centres et des régions dans d'autres domaines de la population hongroise en dehors de ses frontières (voir Papp 1970. 166, 167, 168).

Finalement, il faut tenir aussi compte que certains villages des deux régions peuvent être mutuellment mis en corrélation du point de vue géographique, de l'histoire des localités (proximité du mont, da la rivière, situation dans une vallée ou sur une plaine, appartenance à une région particulière) (Kôszegdoroszló-Hosszúfalu, Dozmat-Csente, Sorokpolány-Felsôlakos).

Du point de vue dialectologique toutes les six localités appartiennent au territoire dialectal hongrois occidental (Imre 1971. 371; Szabó G. 1981. 113). A l'interieur de ce dialecte les villages I-III peuvent être classés dans le type dialectal transdanubien de nord-ouest (Imre 1971. 334-5). De nos jours, les communes IV-VI sont considérées comme appartenant au dialecte hongrois de la région de Mura formant comme isolat une unité specifique (Dávid 1980. 115-6). Mais les contacts de ses différentes parties avec les types de dialecte de Hongrie n'ont pas cessé (la localité IV peut être rapprochée du type de dialecte de Hetés, en temps que les villages V-VI sont proches du type de dialecte de Zala et de Göcsej (voir Imre 1971. 335-6, 337; Szabó J. 1990. 279; Varga J. 1982). Mes recherches ont aussi l'objectif de fournir des données pour l'unité et la diversité de dialecte.

J'ai étudié de chaque point d'enquête le corpus des trois interlocuteurs - choisis par une sélection représentative. J'ai essayé de constituer leur composition selon des critères sociologiques. Dans chaque localité, il y a un interlocuteur de toutes les trois générations (jeunes, d'âge moyen, âgés). La jeune génération est formée par mes interlocuteurs ayant moins de quarante ans. Je compte parmis le interlocutaires d'âge moyen ceux de soixante-cinq ans. Les interlocuteurs au-delà de cet âge représentent la génération âgée. Quant à la répartition par sexe chaque commune est représentée par un homme et deux femmes. En ce qui concerne les professions, dans chaque région elles forment proportionnellement trois groupes: aux alentours de Szombathely la couche des agriculteurs, des femmes au foyer et des employés, aux environs de Lendva le groupe des agriculteurs individueles, des employés et des étudiants.

\subsection{L'instrumentation}

Bien que le corpus collecté des 18 interlocuteurs représentatifs rend possibles aussi des recherches phonétiques et morphologiques, cette fois-ci je n'étudie que leur corpus lexical obtenu par la méthode active, indirecte. Durant l'enquête, j'ai interrogé par périphrase la dénomination des certaines notions. J'ai obtenu ces données en me servant d'un questionnaire de lexique général dont les premières 1-111. positions sont identiques aux questions correspondantes de MNyA. (Szabó G. 1975). En partant de la considération que, dans d'autres cas aussi, je pourrais avoir la possibilité de comparaison avec MNyA., j'ai étudié plus profondément cette partie formée de ces 111 concepts du corpus lexical. Ainsi, j'ai analysé sous plusieurs aspects 2685 données lexicales. J'ai examiné en quelle mesure les réponses des interlocuteurs correspondent au standard hongrois, ou bien à la base dialectale locale, c'est -à-dire quelle en est la 
répartition langue courante-dialecte et selon les types des mots dialectaux. Dans tous les cas j'ai séparé le niveau des lexèmes et les datations des lexèmes. J'ai regroupé également les données par villages, par régions et par les différentes couches sociales. En ce qui suit je ne présente que les principales corrélations des datations des lexèmes à l'aide de la statistique.

\section{L'analyse et le témoignage des données}

\subsection{Les méthodes du dépouillement des données du point de vue du contenu}

J'illustre par des tableaux la répartition du corpus selon les aspects différents. Ces tableaux vont figurer à la fin de mon étude. Dans chacun, je signale tout d'abord la proportion des données de langue courante et de dialecte, après, parmi les données dialectales, je signale la présence des mots dialectaux morphologiques, sémantiques et authentiques. Les symboles $\mathrm{Sz}, \mathrm{L}$ désignent les deux régions, les chiffres I-VI les six points d'enquête. En ce qui suit, je tends également à déduire les conclusions essentielles de chaque tableau.

\subsection{L'analyse statistique des données}

Les tableaux 1.a et $\mathrm{b}$ contiennent les indices du corpus complet analysé ( $\mathrm{Sz}+\mathrm{L})$. La proportion de langue courante-dialecte présentée dans le tableau 1.a correspond aux résultats des recherches similaires effectuées dans la partie occidentale du territoire de la langue hongroise $(29,38 \%-70,61 \%)$. A Szombathely, par exemple, $28 \%$ des données appartenaient à la langue courante, $72 \%$ au dialecte (Szabó G. 1980. 114). A Lickóvadamos du comitat Zala, 27\% des données appartenaient à la langue courante, $73 \%$ au dialecte (Fitos 1982. 138). Dans la région de Felsőőr (Oberwart) en Autriche, on a enregistré $30 \%$ de données de langue courante, $66 \%$ de dialecte et $4 \%$ de langue allemande (Molnár 1986. 162). A Dobronak (Dobrovnik) en Slovénie, le rapport était: $26,27 \%$ des données de langue courante, $73,71 \%$ de dialecte (Szekeres 1972. 24). Selon le témoignage du tableau 1.b, le nombre des lexèmes faisant partie par ailleurs de la langue courante, mais apparaissant sous une forme morphologique de dialecte, est grand $(52,90 \%)$.

La répartition de mes données totalisées entre les régions de Szombathely (Sz) et de Lendva (L) est présentée dans les tableaux 2.a et b. Les données prouvent que la région de Lendva est plus "régionale" que les alentours de Szombathely. Ce phénomène est généré par la différence entre la répartition des mots dialectaux authentiques. Évidemment, ce n'est pas étonnant, en revanche, ce qui est surprenant est le fait que dans la région de Szombathely l'apparition des mots dialectaux sémantiques est plus fréquente.

L'image des deux régions selon les générations est présentée dans les tableaux 3.a et $\mathrm{b}$. Tandis que dans la région de Szombathely la mesure de la régionalité augmente à peu près proportionnellement à partir des jeunes vers les âgés, aux alentours de Lendva 
les gens d'âge moyen sont un peu moins "dialectophiles" que les jeunes. Une des causes de ce phénomène - à côté de la conduite des premiers - peut être le fait que dans l'isolat de langue hongroise de Slovénie les jeunes vivent relativement longtemps dans l'entourage des âgés, et que cela ait une influence importante sur leur régionalisme, surtout dans les mots dialectaux morphologiques et dans la prononciation dialectale. En revanche, selon les données de la région de Szombathely, ici ce sont les gens d'âge moyen qui sont plus proches des âgés, bien que le nombre des mots dialectaux sémantiques de la génération âgée puisse être significatif.

La recherche selon les sexes qui s'est basée seulement sur la matière des âgés et de la génération d'âge moyen étant donné que la répartition homme-femme est proportionnelle dans leurs cas, a eu pour résultat les indices figurant dans les tableaux 4.a et $b$. De ce point de vue, les deux régions ont une grande ressemblance. Dans toutes les deux, c'étaient les femmes qui ont fourni plus de variations de lexèmes. Dans toutes les régions, ce sont elles qui se manifestent plus régionales, ce qui résulte de l'emploi plus fréquent des mots dialectaux sémantiques et morphologiques (aux alentours de Szombathely les femmes dépassent les hommes dans le groupe des mots dialectaux sémantiques, dans la région de Lendva dans le groupe des mots dialectaux sémantiques et morphologiques). Par contre, les représentants du sexe fort viennent en tête dans le cas des mots dialectaux authentiques, donc dans le groupe des formes plus archä̈ques (aux environs de Lendva dans une proportion plus grande).

En examinant le corpus du point de vue des professions, on arrive aux observations enregistrées dans les tableaux 5.a et b. En général, on peut considérer valable l'affirmation selon laquelle l'érudition est accompagnée par l'usage de la langue courante. Cette théorie est approuvée dans toutes les deux régions par la proportion agriculteurs-agriculteurs individuels et des employés, c'est-à-dire que simultanément avec l'augmentation de la formation, le nombre des mots dialectaux authentiques diminue. Les données suivantes montrent une différenciation, car, par exemple, dans la région de Szombathely ce sont les femmes au foyer qui viennent en tête dans le régionalisme, tandis que dans les environs de Lendva, l'emploi du dialecte est plus caractéristique pour les étudiants que pour les employés. Dans le premier cas, le résultat est lié à la fréquence des mots dialectaux morphologiques. Cette remarque coïncide avec les observations selon lesquelles le niveau culturel n'a pas nécessairement d'influence directe sur la prononciation dialectale. Naturellement, la conjucture de la situation de ces endroits peut aussi avoir d'autres raisons extérieures. Par exemple, le fait que les femmes au foyer de la région de Szombathely ont à peu près les mêmes conditions de vie que les agriculteurs. Les étudiants originaires de la région de Lendva font leurs études à Maribor, donc dans un milieu linguistique tout slovène; déracinés de leur milieu d'origine et dans un fort isolat linguistique ils conservent le caractère dialectal.

Ces conditions offrent la possibilité de continuer à analyser d'autres corrélations, puisque la communication linguistique, la navette et d'autres facteurs aussi peuvent avoir une influence sur les manifestations linguistiques. Les détails de tous ces 
problèmes, de même que l'illustration de ce qu'on vient de présenter par un corpus lexical concret dépasseraient les cadres de ma présente étude (je présente quand même un échantillon dans l'annexe 2.). J'espère d'avoir encore une autre occasion d'en parler. Pour l'instant je me limite à faire quelques remarques récapitulatives.

\section{Conclusion}

En comparaison avec les recherches complexes ayant lieu dans la région occidentale du territoire linguistique hongrois mon étude peut être considérée comme une microanalyse ou on peut la prendre comme en faisant partie (voir l'introduction). Mais elle tend à esquisser d'une manière nuancée la situation linguistique par l'application comparative des aspects de géographie linguistique et sociolinguistiques.

Sans répeter ce que je viens de présenter je me contente seulement de souligner que dans les phénomènes présentés les mots dialectaux morphologiques avaient plusieurs fois un rôle signifiant. Ils font plus que la moitié de la couche dialectale du corpus séparé de la région de Szombathely ayant un caracère plus proche de la langue courante et dans celui de la région de Lendva ayant un caractère plus régional. Parmi les différentes générations ce type du mot dialectal apparaît de manière éclatante aux alentours de Szombathely, tout comme dans la région de Lendva dans la plus grande proportion chez les jeunes. Ils influencent également la régionalité de la datation de lexèmes chez les femmes, surtout de la région de Lendva. Ils sont présents chez les gens cultivés aussi, de plus il paraît que les étudiants hongrois en Slovénie les acceptent consciemment.

Bien que ces remarques soient associées hors de leur contexte, et par conséquent, leur intensification ne peut être considérée que relative, ces phénomènes ne peuvent pas être négligés. Il paraît que dans le mouvement du lexique synchrone, dans les relations entre les couches, les mots dialectaux jouent un rôle intense. D'une part ils peuvent représenter un milieu médiateur vers la langue courante, d'autre part ils peuvent constituer un point d'appui dans la conservation des valeurs dialectales. De cette manière ils peuvent servir à la confrontation saine, à l'appréciation réelle des variations de la langue hongroise. Dans les contextes au-delà des frontières ils peuvent contribuer à la continuité de la langue hongroise.

Finalement, par la totalité de mes recherches, je voudrais contribuer à la réalisation de ces objectifs. 


\section{Les tableaux}

Tableau 1.a

La répartition des éléments de langue courante-dialectaux sur l'ensemble du corpus

\begin{tabular}{|l|c|c|c|}
\hline & Total & Langue courante & Dialectal \\
\hline Sz + L & 2685 & 789 & 1896 \\
$(\mathrm{I}+\mathrm{II}+\mathrm{III}+\mathrm{IV}+\mathrm{V}+\mathrm{VI})$. & $100 \%$ & $29,38 \%$ & $70,61 \%$ \\
\hline
\end{tabular}

Tableau 1.b

La répartition du corpus dialectal selon les catégories de mots dialectaux

\begin{tabular}{|l|c|c|c|c|}
\hline & Dialectal & Morphologique & Sémantique & Authentique \\
\hline $\mathrm{Sz}+\mathrm{L}$ & 1896 & 1003 & 362 & 531 \\
$(\mathrm{I}+\mathrm{II}+\mathrm{III}+\mathrm{IV}+\mathrm{V}+\mathrm{VI})$. & $100 \%$ & $52,90 \%$ & $19,09 \%$ & $28,00 \%$ \\
\hline
\end{tabular}

Tableau 2.a

La répartition des éléments de langue courante-dialectaux selon les régions

\begin{tabular}{|l|c|c|c|}
\hline & Total & Langue courante & Dialectal \\
\hline $\mathrm{Sz}$ & 1482 & 492 & 990 \\
$(\mathrm{I}+\mathrm{II}+\mathrm{III}+\mathrm{IV}+\mathrm{V}+\mathrm{VI})$. & $100 \%$ & $33,19 \%$ & $66,80 \%$ \\
$\mathrm{~L}$ & 1203 & 297 & 906 \\
$(\mathrm{I}+\mathrm{II}+\mathrm{III}+\mathrm{IV}+\mathrm{V}+\mathrm{VI})$. & $100 \%$ & $24,68 \%$ & $75,31 \%$ \\
\hline
\end{tabular}

Tableau 2.b

La répartition des catégories de mots dialectaux selon les régions

\begin{tabular}{|l|c|c|c|c|}
\hline & Dialectal & Morphologique & Sémantique & Authentique \\
\hline $\mathrm{Sz}$ & 990 & 527 & 204 & 259 \\
$(\mathrm{I}+\mathrm{II}+\mathrm{III}+\mathrm{IV}+\mathrm{V}+\mathrm{VI})$. & $100 \%$ & 53,23 & $20,60 \%$ & $26,16 \%$ \\
$\mathrm{~L}$ & 906 & 476 & 158 & 272 \\
$(\mathrm{I}+\mathrm{II}+\mathrm{III}+\mathrm{IV}+\mathrm{V}+\mathrm{VI})$. & $100 \%$ & $52,53 \%$ & $17,43 \%$ & $30,02 \%$ \\
\hline
\end{tabular}


Tableau 3.a

La répartition des éléments de langue courante-dialectaux selon les générations

\begin{tabular}{|l|c|c|c|}
\hline & Total & Langue courante & Dialectal \\
\hline Sz (I+II+III.) & & & \\
Jeunes & 471 & 204 & 267 \\
& $100 \%$ & $43,31 \%$ & $56,68 \%$ \\
D'âge moyen & 512 & 154 & 358 \\
& $100 \%$ & $30,07 \%$ & $69,92 \%$ \\
Âgés & 499 & 134 & 365 \\
& $100 \%$ & $26,85 \%$ & $73,14 \%$ \\
\hline L (IV+V+VI.) & & & \\
Jeunes & 466 & 121 & 345 \\
& $100 \%$ & $25,96 \%$ & $74,03 \%$ \\
D'âge moyen & 391 & 107 & 284 \\
& $100 \%$ & $27,36 \%$ & $72,63 \%$ \\
Âgés & 346 & 69 & 277 \\
& $100 \%$ & $19,94 \%$ & $80,05 \%$ \\
\hline
\end{tabular}

Tableau 3.b

La répartition des catégories de mots dialectaux selon les générations

\begin{tabular}{|l|c|c|c|c|}
\hline & Dialectal & Morphologique & Sémantique & Authentique \\
\hline Sz (I+II+III.) & & & & \\
Jeunes & 267 & 147 & 50 & 70 \\
D'âge moyen & $100 \%$ & $55,05 \%$ & $18,72 \%$ & 26,21 \\
& 358 & 193 & 68 & 97 \\
Âgés & $100 \%$ & $53,91 \%$ & $18,99 \%$ & $27,09 \%$ \\
& 365 & 187 & 86 & 92 \\
\hline L (IV+V+VI.) & $100 \%$ & $51,23 \%$ & $23,56 \%$ & $25,20 \%$ \\
Jeunes & & & & \\
D'âge moyen & 345 & 202 & 52 & 91 \\
& $100 \%$ & $58,55 \%$ & $15,07 \%$ & $26,37 \%$ \\
Âgés & 284 & 144 & 51 & 89 \\
& $100 \%$ & $50,70 \%$ & $17,95 \%$ & $31,33 \%$ \\
& 277 & 130 & 55 & 92 \\
& $100 \%$ & $46,93 \%$ & $19,85 \%$ & $33,12 \%$ \\
\hline
\end{tabular}


Tableau 4.a

La répartition des éléments de langue courante-dialectaux selon des sexes

\begin{tabular}{|l|c|c|c|}
\hline & Total & Langue courante & Dialectal \\
\hline Sz (I+II+III.) & & & \\
Femmes & 546 & 147 & 399 \\
& $100 \%$ & $26,92 \%$ & $73,07 \%$ \\
Hommes & 465 & 141 & 324 \\
& $100 \%$ & $30,32 \%$ & $69,67 \%$ \\
\hline L (IV+V+VI.) & & & \\
Femmes & 389 & 91 & 298 \\
& $100 \%$ & $23,39 \%$ & $76,60 \%$ \\
Hommes & 351 & 108 & 243 \\
& $100 \%$ & $30,76 \%$ & $69,23 \%$ \\
\hline
\end{tabular}

Tableau 4.b

La répartition des catégories de mots dialectaux selon les sexes

\begin{tabular}{|l|c|c|c|c|}
\hline & Total & Langue courante & Dialectal & Authentique \\
\hline Sz (I+II+III.) & & & & \\
Femmes & 399 & 206 & 92 & 101 \\
& $100 \%$ & $51,62 \%$ & $23,05 \%$ & $25,31 \%$ \\
Hommes & 324 & 174 & 62 & 88 \\
& $100 \%$ & $53,70 \%$ & $19,13 \%$ & $27,16 \%$ \\
\hline L (IV+V+VI.) & & & & \\
Femmes & 298 & 154 & 56 & 88 \\
& $100 \%$ & $51,67 \%$ & $18,79 \%$ & $29,53 \%$ \\
Hommes & 243 & 109 & 44 & 90 \\
& $100 \%$ & $44,85 \%$ & $18,10 \%$ & $37,03 \%$ \\
\hline
\end{tabular}


Tableau 5.a

La répartition des éléments de langue courante-dialectaux selon les catégories de profession

\begin{tabular}{|l|c|c|c|}
\hline & Total & Langue courante & Dialectal \\
\hline Sz (I+II+III.) & & & \\
Agriculteurs & 465 & 141 & 324 \\
Femmes au foyer & $100 \%$ & $30,32 \%$ & $69,27 \%$ \\
& 546 & 147 & 399 \\
Employés & $100 \%$ & $26,92 \%$ & $73,07 \%$ \\
& 471 & 204 & 267 \\
\hline L (IV+V+VI.) & $100 \%$ & $43,31 \%$ & $56,68 \%$ \\
Agriculteurs individuels & 346 & & \\
& $100 \%$ & 69 & 277 \\
Employés & 391 & $19,94 \%$ & $80,05 \%$ \\
Étudiants & $100 \%$ & 107 & 284 \\
& 466 & $27,36 \%$ & $72,63 \%$ \\
\hline
\end{tabular}

Tableau 5.b

La répartition des catégories de mots dialectaux selon les catégories de profession

\begin{tabular}{|l|c|c|c|c|}
\hline & Dialectal & Morphologique & Sémantique & Authentique \\
\hline Sz (I+II+III.) & & & & \\
Agriculteurs & 324 & 174 & 62 & 88 \\
Femmes au foyer & $100 \%$ & $53,70 \%$ & $19,13 \%$ & $27,16 \%$ \\
& 399 & 206 & 92 & 101 \\
Employés & $100 \%$ & $51,62 \%$ & $23,05 \%$ & $25,31 \%$ \\
& 267 & 147 & 50 & 70 \\
\hline L (IV+V+VI.) & $100 \%$ & $55,05 \%$ & $18,72 \%$ & $26,21 \%$ \\
Agriculteurs individuels & 277 & & & \\
& $100 \%$ & 130 & 55 & 92 \\
Employés & 284 & 144 & $19,85 \%$ & $33,21 \%$ \\
& $100 \%$ & $50,70 \%$ & 51 & 89 \\
Étudiants & 345 & 202 & $17,95 \%$ & $31,33 \%$ \\
& $100 \%$ & $58,55 \%$ & $15,07 \%$ & $26,37 \%$ \\
\hline
\end{tabular}




\section{La bibliographie}

BDTF TudKözl. I-II., IV-V. Molnár Károly (réd.): A szombathelỷi Berzsenyi Dániel Tanárképzó Fớiskola Tudományos Közleményei (Szombathely) I (1978), II (1980), IV (1984), V (1986).

BDTF TudKözl. VI. Szabó Géza (réd.): A szombathelyi Berzsenyi Dániel Tanárképzô Fôiiskola Tudományos Közleményei (Szombathely) VI. Nyelvtudomány (1990).

Dávid András 1980. Nyelv és varázs. Tankönyvikiadó, Budapest.

Deme László (réd.) 1968. Gyújtési útmutató a "Városunk köznyelvi kiejtése" címen hirdetett középiskolás pályázathoz. Magyartanítás (Budapest) 1, 2-17.

Deme László 1995: Nyelvünk többközpontúságának kérdéséhez. Magyar Nyelvốr (Budapest) $119,357-65$.

DialSzimp. I. Szabó Géza-Molnár Zoltán (réd.): [I.] Dialektológiai Szimpozion. VEAB (Veszprém), 1982.

DialSzimp. II. Szabó Géza (réd.): II. Dialektológiai Szimpozion. VEAB (Veszprém), 1990.

Fitos Amália 1982. Szókészleti vizsgálatok Lickóvadamos nyelvjárásában. $=\mathrm{A}$ XV. OTDK nívódíjas pályamunkái. II., T. I. I., Budapest, 137-40.

FonOpisi Nedim Filipovič (réd.): Fonološki opisi srpskohrvatskih/hrvatskosipskih, slovenačkih i makedonskih govora obuhvaćenih opšteslovanskim lingvističkim atlasom. Sarajevo, 1981.

Goebl, Hans 1988. Il posto dialettometrico che spetta ai punti-AIS 338 (Adorgnano, Friuli), 398 (Dignano/Vodjan, Istria) e 367 (Grado, Friuli). Linguistica (Ljubljana) XXVIII, 75-103.

Imre Samu 1971. A mai magyar nyelvjárások rendszere. Akadémiai K., Budapest.

Jakopin, Franc 1989. Pokrajinski tipi slovenskih priimkov. = XXV. seminar slovenskega jezika, literature in kulture. Ljubljana, 61-5.

Jernej, Josip 1988. Considerazioni sui problemi sociolinguistici nelle regioni dell' Alpe-Adria. Linguistica (Ljubljana) XXVIII, 47-8.

Kálmán Béla 1983. Nyelvjáráskutatás Finnországban. Magyar Nyelv (Budapest) LXXIX, 91-7.

Kálmán Béla 1986. Az Európai Nyelvatlasz. Magyar Nyelvốr (Budapest) 110, 111-7.

Kiss Jenô 1987. Erốvonalak az európai dialektológiában. Magyar Nyelv (Budapest) LXXXIII, 140-5.

Korotkova, T. Sz. 1988. Zametki o sovremennoj oblastnoj lexikografiji. Russkaja Reč (Moskva) 1, 52-9.

Kovačec, August 1992. Éléments italiens du lexique istroroumain. Linguistica (Ljubljana) XXXII, 159-75.

Križman, Mirko 1989. Jezik kot socialni pojav. Maribor, 127-39, 223-40.

KSH 1990. évi népszámlálás. Elốzetes adatok. Központi Statisztikai Hivatal, Budapest, 1990.

Lanstyák István 1995. A nyelvek többközpontúságának néhány kérdéséről. Magyar Nyelvőr (Budapest) 119, 213-36.

Logar, Tine 1975. Izvenjezikovni vzroki za dialektizacijo slovenskega jezika. $=\mathrm{XI}$. seminar slovenskega jezika, literature in kulture. Ljubljana, 7-11. 
Logar, Tine 1989. Slovenski lingvistični atlas. = XXV. seminar slovenskega jezika, literature in kulture. Ljubljana, 9-22.

MNyA. Deme László-Imre Samu (réd. avec la collaboration de la collectivité de travail): A magyar nyelvjárások atlasza I-IV. Akadémiai K., Budapest, 1968-1978.

MNyAElmMódsz. Deme László-Imre Samu (réd.): A magyar nyelvjárások atlaszának elméleti-módszertani kérdései. Akadémiai K., Budapest, 1975.

MNyRétegz. Kiss Jenô-Szűts László (réd.): A magyar nyelv rétegző́dése. Akadémiai K, Budapest 1988. 39-49, 79-85, 108-19, 297-307, 402-8, 484-92, 554-65, 628-37, 736-51, 766-79, 821-31, 906-13, 976-87, 1029-40, 1054-63.

Molnár Zoltán Miklós 1980. Vizsgálatok a szombathelyi főiskolások beszédhanghasználatának köréböl. = BDTF TudKözl. II, 71-86.

Molnár Zoltán Miklós 1986. Élổ nyelvi megfigyelések Felsôôr vidékén. = Szalay László (red.): Hungarológiai napok. Szombathely, 161-5.

Molnár Zoltán Miklós 1989. Szombathely környéki földrajzi nevek vallomásai egy elónyelvi anyag tükrében. $=$ Balogh Lajos-Ördög Ferenc (réd.): Névtudomány és múvelödéstörténet. Zalaegerszeg, 146-50.

Molnár Zoltán Miklós 1990. Nyelvföldrajzi-nyelvszociológiai vizsgálatok néhány kérdése Nyugat-Dunántúlon. = BDTF TudKözl. VI, 43-52.

Molnár Zoltán Miklós 1991. Muravidéki élőnyelvi adatok A magyar nyelvjárások atlasza kóti anyagának tükrében. = Hajdú Mihály-Kiss Jenó (réd.): Emlékkönyv Benkố Loránd hetvenedik születésnapjára. Budapest, 467-71.

Nečak Lük, Albina 1992. A muravidéki kétnyelvŭ iskolák tanulóinak kétnyelvü kommunikációs készségéről. = Győri-Nagy Sándor-Kelemen Janka (réd.): Kétnyelvúség a Kárpátmedencében II., Budapest, 56-67.

Papp, György 1990. A bácskai nyelvjárások változása és a köznyelvi norma. = Balogh Lajos-Kontra Miklós (réd.): Élốnyelvi tanulmányok. MTA Nyelvtudományi Intézet, Budapest, 164-8.

Ramovš, Fran 1931. Dialektološka karta slovenskega jezika. Ljubljana.

SLA Slovenski lingvistični atlas. = Danica Korošec: Kebeljski govor. Maribor, 1991. 43-172.

Szabó Géza 1975. Szókészleti kérdőfüzet. Szombathely.

Szabó Géza 1976. A nyelvjáráskutatás jelentôségérôl és lehetôségeirôl Nyugat-Dunántúlon. Vasi Szemle (Szombathely) 3, 400-23.

Szabó Géza 1980. Regionális szókészleti kutatás Szombathelyen. Vasi Szemle (Szombathely) 1, 104-39.

Szabó Géza 1981. A magyar nyelvjárások. Tankönyvkiadó, Budapest.

Szabó József 1990. Magyarországi és jugoszláviai magyar nyelvjárásszigetek. Békéscsaba, Kecskemét, Szeged.

Szekeres Ilona 1992. Nyelvszociológiai vizsgálatok Dobronak élổnyelvében. maribor.

Szentmihályi Imre 1977. Hetés és Lendva-vidék néprajzi sajátosságai (Zalai Gyújtemény 7.). Zalaegerszeg.

Szépe György 1985. Anyanyelv, nyelvi politika, oktatás. Magyar Nyelv (Budapest) LXXXI, 267-79.

Tesnière, Lucien 1925. Atlas linguistique pour servir a l'étude du duel en slovène. Travaux publiés par l'Institut d'études slaves: atlas hors série annexe au tome III, Paris. 
Toporišič, Jože 1991. Slovenska slovnica. Maribor, 10-30, 666-84.

Varga József 1982. A hetési nyelvjárás. = Penavin Olga (réd.): Jugoszláviai magyar nyelvjárások. Újvidék, 177-9.

Varga József 1985. A murántúli falvak ragadványnevei. Kézirat.

Varga Sándor 1992. A lendvai plébánia(templom) történetéből. Hazánk, Gyốr.

Vas megye, 1979. Vas megye térképe. Kartográfiai Vállalat, Budapest. ${ }^{3}$

Zbornik, 1981. Zbornik občine Lendava - Lendva község közleménye. LendavaLendva.

Zorko, Zinka 1989a. Narečna podoba mariborskega predmestja. = Časopis za zgodovino in narodopisje (Maribor) 2, 139-4.7.

Zorko, Zinka 1989b. Framski govor. = Znanstvena revija (Maribor) 2, 133-45.

Annexe no. 1

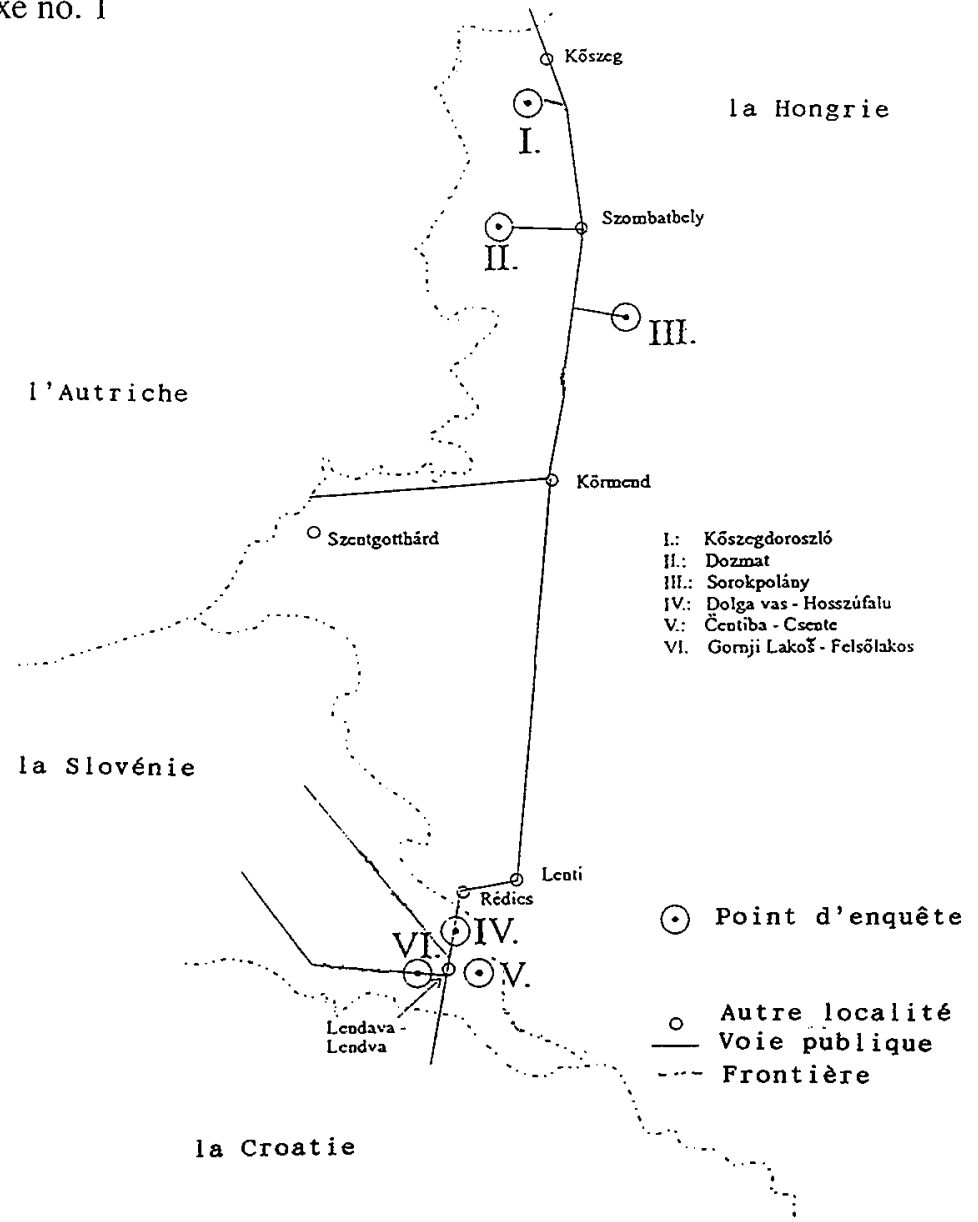

In: (la carte de) Hongrie. La Fédération des bureaux de tourisme. Sans lieu et année. 
Annexe no. 2

Échantillon du corpus lexical collecté aux environs de Szombathely et de Lendva

Question no. 1, kalász žépi’ (mot courant): Quel est la dénomination de la partie supérieure des céréales où se trouvent les graines? (Quand il mûrit, il s'incline.) (La question no. 7 de MNyA.)

La région de Szombathely L'interlocuteur:

I. 1. kalász, gabonafej jeune, femme, couturière

2. fej, fejë, kalász d'âge moyen, homme, agriculteur

3. kalász, buzakalász âgée, femme, femme de foyer

II. 4. kalász jeune, femme, agriculteur

5. fej, buzafej d'âge moyen, femme, femme au foyer

6. kalász, fej âgé, homme, agriculteur

III. 7. kalász

jeune, femme, institutrice

8. kalász

9. polva

d'âge moyen, femme, femme au foyer

âgé, homme, agriculteur

La région de Lendva

IV. 10. kalász, fej

jeune, femme, étudiante

11. buzafej

d'âge moyen, homme, agriculteur individuel

12. a gabona feje

âgé, homme, agriculteur individuel

V. 13. kălász, fej

jeune, femme, étudiante

14. buzafej

d'âge moyen, femme, institutrice

15. fej

âgé, homme, ouvrier

VI. 16. kalász, buzafej

jeune, femme, étudiante

17. feje, buzafej

d'âge moyen, femme, vendeuse

18. kalász

âgée, femme, agriculteur individuel

Povzetek

PRIMERJALNA RAZISKAVA BESEDIŠČA ROJENIH GOVORCEV MADŽARSKEGA JEZIKA NA DVEH

OBMOČJIH

Prispevek je izvleček iz širše raziskave, $v$ kateri avtor razčlenjuje in primerja družbeno zasnovane govorne variacije $v$ dveh okoljih, ki sta primerljivi glede na določene zemljepisne (razgibanost zemljepisnega območja) ter družbene značilnosti (razmerje urbano : ruralno). Cilj raziskave je prepoznati in primerjati zvrstno pripadnost besedišča, ki ga posedujejo rojeni govorci madžarskega jezika $v$ treh naseljih zahodne Madžarske v okolici Sombotelja (Kổszegdoroszló, Dozmat in Sorokpolány) in v treh naseljih na narodnostno mešanem območju Prekmurja v Sloveniji v okolici Lendave (Dolga vas/Hosszúfalu, Čentiba/Csente in Gornji Lakoš/Felsőlakos). Izbrana raziskovalna metoda je kombinacija metodoloških prijemov s področja geolingvistike ter mikrosociolingvistike. Instrumentarij se zgleduje po dialektološki raziskavi Inštituta za jezik Madžarske 
akademije znanosti. Vzorec je ustrezno razslojen in upošteva spol, starost in socialni status informantov. Avtor ugotavlja, da navedene neodvisne spremenljivke $v$ obeh okoljih vplivajo na številčno razmerje med besedami iz narečne in splošnopogovorne baze: narečne besede so pogostejše pri ženskah, starejših in manj izobraženih. Zanimivo je, da so te povezave manj izražene med rojenimi govorci madžarskega jezika v Sloveniji. Avtor ugotavlja, da v Prekmurju narečna oblika prevladuje, višje izobraženi govorci jo po njegovem zavestno postavljajo v ospredje. 